Pacific Journal of Mathematics

INVARIANT SUBSPACES AND PROJECTIVE 


\title{
INVARIANT SUBSPACES AND PROJECTIVE REPRESENTATIONS
}

\author{
KEITH YALE
}

\begin{abstract}
Let $\Gamma$ be a subgroup of the real line $R$ with the discrete topology, and let $G$ be its compact dual group. This paper shows the existence of a (nontrivial) simply invariant subspace of $L^{2}(G)$ which is not of the form $\varphi H^{2}(G)$ provided $\Gamma$ contains at least two rationally independent elements. The proof relies heavily on the existence of a nontrivial local projective representation of the two-dimensional torus.
\end{abstract}

Helson and Lowdenslager [4] showed the existence of a simply invariant subspace not of the form $\varphi H^{2}(G)$ in case $I^{\top}$ contains an infinite set of rationally linearly independent elements. We use the correspondence introduced in [4] between simply invariant subspaces and cocycles but in contrast to [4] we use nontrivial local projective multipliers to show that the appropriate cohomology group is nontrivial.

The connection between invariant subspaces and cocycles is discussed in $\S 2$ and in $\S 3$ we will give a quotient group argument which allows us to reduce the general problem to its specialization on the two-dimensional torus. Sections 4 and 5 relate the notion of projective representation with a cocycle and it is shown that a nontrivial projective representation gives rise to a cocycle whose corresponding subspace is not of the form $\varphi H^{2}(G)$.

2. Preliminaries. Let $G$ be an arbitrary locally compact Abelian group dual to $\Gamma$ and let $\Lambda$ be a continuous one-parameter subgroup of $G$ which we also denote by $\left\{e_{t} \mid t\right.$ in $\left.R\right\}$. Haar measure in $G$ will be denoted by $d x$ and will be normalized to have total mass one in case $G$ is compact. As usual, a.e. $(x)$ means for all but a set of Haar measure zero. A (Borel) function $\varphi$ on $G$ is said to be unitary in case $\varphi(x)$ has modulus one a.e. $(x)$.

Definition. A function $A$ on $A \times G$ is said to be a cocycle on $G$ in case:

$$
\begin{gathered}
A\left(e_{t}, \quad\right) \text { is a unitary function for each } e_{t} \text { in } \Lambda, \\
A\left(e_{t}+e_{u}, x\right)=A\left(e_{u}, x\right) A\left(e_{t}, x-e_{u}\right) \text { for all } e_{t}, e_{u} \text { in } \Lambda
\end{gathered}
$$

and a.e. $(x)$, and

$$
A \text { is strongly continuous in the sense that } A\left(e_{t}, \quad\right) f
$$

is a continuous function from $R$ into $L^{2}=L^{2}(G)$ for $f$ in $L^{2}$. 
Cocycles of the form

$$
A\left(e_{t}, x\right)=\varphi(x) / \varphi\left(x-e_{t}\right), \text { all } e_{t} \text { in } \Lambda \text {, a.e. }(x)
$$

for some unitary function $\varphi$ are called coboundaries. We will frequently denote $A\left(e_{t}, x\right)$ by $A(t, x)$.

If $\lambda$ is in $\Gamma$ we let $\chi_{\lambda}$ be the character on $G$ defined by $\chi_{\lambda}(x)=$ $x(\lambda)$ for all $x$ in $G$; the corresponding unitary representation $V_{0}$ of $\Gamma$ is given by

$$
V_{0}(\lambda) f(x)=\chi_{\lambda}(x) f(x)
$$

for all $f$ in $L^{2}$. Any bounded operator on $L^{2}$ which commutes with all the $V_{0}(\lambda)$ is necessarily a multiplication by a function in $L^{\infty}$. Let $U_{0}$ be the unitary representation of $G$ defined by

$$
U_{0}(x) f(y)=f(y-x)
$$

for all $f$ in $L^{2}$.

For the remainder of this section we will let $\Gamma$ be a subgroup of the real line $R$. Let $G$ be the compact Abelian group dual to the discretely topologized $\Gamma$. A closed subspace $\mathscr{C l}$ of $L^{2}$ is said to be simply invariant in case $V_{0}(\lambda) \mathscr{M} \subseteq \mathscr{M}$ if and only if $\lambda \geqq 0$. The Hardy space $H^{2}$ consists of those functions $f$ in $L^{2}$ whose Fourier transforms $\hat{f}(\lambda)=\int \chi_{-\lambda}(x) f(x) d x$ vanish for $\lambda<0$. Subspaces of the form $\mathscr{L}=\varphi H^{2}=\left\{\varphi f: f\right.$ in $\left.H^{2}\right\}$ where $\varphi$ is a unitary function are simply invariant and in the case where $G$ is a circle all simply invariant subspaces are of this form.

In order to avoid the rather special circle group we will henceforth suppose that $\Gamma$ is dense in $R$. The characters $e_{t}$ defined by $e_{t}(\lambda)=$ $\exp (i t \lambda)$ are distinct and provide a continuous one-parameter dense subgroup $A$ of $G$. A correspondence is exhibited in [3,4] between simply invariant subspaces $\mathscr{C}$ (suitably normalized) and cocycles $A$ in such a way that $\mathscr{C}=\varphi H^{2}$ if and only if $A$ is the coboundary (2.4). We therefore wish to construct cocycles which are not coboundaries.

If $A$ is a coboundary then $A$ can be extended from $A \times G$ to $G \times G$ so that (2.4) remains valid with $t$ replaced by an arbitrary $y$ in $G$ and conversely. Moreover, the multiplication operator $A(y, \quad)$ is the strong operator limit of a sequence $A\left(t_{n}, \quad\right)$ where $e_{i_{n}}$ tends to $y$ in $G$; this observation will be useful later. Equivalently, $A$ is a coboundary if and only if the unitary representation $U(t)=A(t, \quad) U_{0}(t)$ can be extended from $\Lambda$ to a (strongly continuous) unitary representation of $G$. A cocycle was constructed in [4] (in case $\Gamma$ is suitably large) for which the unitary representation did not extend to $G$. However, it is conceivable that $U(t)$ might extend to a (local) projective representation of $G$; this idea is turned around and will be used to extract cocycles 
from projective representations.

There is a superficial answer to our problem in case $\Gamma$ is not all of $R$ for then there are trivial cocycles which are not coboundaries. For example, let $A(t, x)=\exp (-i t \lambda)$ for some fixed real $\lambda$ not in $\Gamma$. If $\lambda$ were in $\Gamma$ then $A$ would be the coboundary with unitary function $\chi_{\lambda}$ but with $\lambda$ not in $\Gamma$ there is no unitary function $\varphi$ such that $\exp (-i t \lambda)=\varphi(x) / \varphi\left(x-e_{t}\right)$. Conversely, if $A$ is a cocycle which is constant a.e. $(x)$ for each $t$ (the null set depending upon $t$ ), then $A(t, x)=\exp (-i t \lambda)$, a.e. $(x)$ for some fixed $\lambda$ in $R$. We will call cocycles of this form constant cocycles. Consequently the nontrivial problem [3, p. 149] is to find cocycles which are not products of constant cocycles and coboundaries.

The cocycles defined in [3] were measurable functions on $A \times G$ but we will have no need for cocycles to be product measurable. Anyway, one can pass from one version to another [3, p. 145], [2]. Also we have departed from [3] by making an insignificant sign change in our definition of cocycle.

3. Reduction to the torus, Suppose that $\Gamma_{0} \subseteq \Gamma$ are subgroups of the discrete real line and let $G_{0}$ and $G$ be their compact dual groups. To each cocycle $A_{0}$ on $G_{0}$ we will associate a cocycle $A$ on $G$ in such a way that if $A$ is the product of a constant cocycle and a coboundary then so is $A_{0}$. Since the two-dimensional torus $T^{2}$ is dual to the group of lattice points $Z^{2}$ and $Z^{2}$ is isomorphic to a subgroup $\Gamma_{0} \subseteq \Gamma$ of any group $\Gamma \leqq R$ with at least two independent elements it will be sufficient to construct a cocycle on $T^{2}$ which is not the product of a constant cocycle and a coboundary.

Define a closed subgroup $H=\left\{x\right.$ in $G \mid \chi_{\lambda}(x)=1$ for all $\lambda$ in $\left.\Gamma_{0}\right\}$ of $G$ so that $G_{0}$ can be identified with $G / H$. Let $\pi$ be the usual quotient map from $G$ onto $G / H$ and let $e_{t}$ and $\varepsilon_{t}$ be the previously defined oneparameter groups $\Lambda$ and $\Lambda_{0}$ in $G$ and $G_{0}$. One can verify $\pi\left(e_{t}\right)=\varepsilon_{t}$ by noting that $\varepsilon_{t}$ is the restriction of $e_{t}$ from $\Lambda$ to $\Lambda_{0}$.

If $A_{0}$ is a cocycle on $G_{0}$ we define a cocycle $A$ on $G$ by

$$
A\left(e_{t}, x\right)=A_{0}\left(\varepsilon_{t}, \pi(x)\right)
$$

for all $\left(e_{t}, x\right)$ in $\Lambda \times G$.

For each $t$ in $R$ the measurable function $A\left(e_{t}, \quad\right)$ on $G$ is certainly unitary because $\pi^{-1}(S)$ is a null set in $G$ whenever $S$ is a null set in $G / H$. The cocycle identity (2.2) is easy enough to verify with the aid of $\pi\left(e_{t}\right)=\varepsilon_{t}$ so all that remains is the strong continuity.

Let the Haar measures $d x$ and $d x_{0}$ in $G$ and $G / H$ both be normalized to have total mass one. There is a normalization for the Haar measure $d \xi$ on $H$ such that 


$$
\int_{G} f(x) d x=\int_{G / H}\left(\int_{H} f(x+\xi) d \xi\right) d x_{0}
$$

for all $f$ in $L^{1}(G)$.

Let $f$ be in $L^{2}(G)$ and put

$$
g\left(x_{0}\right)=\int_{H}|f(x+\xi)|^{2} d \xi
$$

where $x_{0}=\pi(x)$. A straight-forward computation with (3.2) shows that $A\left(e_{t},\right) f$ moves continuously in $L^{2}(G)$ as $t$ varies because $A_{0}\left(e_{t},\right) \sqrt{g}$ moves continuously in $L^{2}(G / H)$.

THEOREM. If $A$ is the product of a constant cocycle and a coboundary then so is $A_{0}$.

Proof. For some constant cocycle $C$ and some unitary function $\varphi$ on $G$ we have

$$
C(t) A(t, x)=\varphi(x) / \varphi\left(x-e_{t}\right)
$$

for each real $t$ and almost all $x$.

It is advantageous to normalize by choosing $\lambda$ in $R$ such that $\int \chi_{\lambda}(x) \varphi(x) d x$ does not vanish and putting $\psi=\chi_{\lambda} \varphi$. The cocycle $B=$ $\chi_{2} C A$ is really the coboundary.

$$
B(t, x)=\psi(x) / \psi\left(x-e_{t}\right)
$$

and we have $B_{0}=\chi_{\lambda} C A_{0}$. Consequently it is sufficient to show that $B_{0}$ is a coboundary and we will do this by arguing that $\psi$ must be constant on cosets of $H$.

Since $B(t, x)=B_{0}(t, \pi(x))$ it follows that $B(t, x)=B(t, x+h)$ for all real $t$ and all $(x, h)$ in $G \times H$. Now the coboundary $B$ can be extended to $G \times G$ and, in fact, $B(y$,$) is a limit in L^{2}(G)$ of a sequence $B\left(t_{n}\right.$, ) where $e_{t_{n}}$ goes to $y$ in $G$. Therefore, passing to a subsequence if necessary, $B\left(t_{n}, x\right)$ tends to $B(y, x)$ for almost all $x$ and we can conclude

$$
B(y, x)=B(y, x+h)
$$

for all $y$ in $G, h$ in $H$ and almost all $x$ in $G$.

From (3.3) (valid now for $t$ replaced by any element in $G$ ) and (3.4) we have

$$
\psi(x+\xi)=B(h, x) \psi(x+\xi-h)
$$

for every $\xi$ in $H$ and almost all $x$ in $G$. Integrating this last expression with respect to Haar measure $d \xi$ on $H$ we find 


$$
\int_{H} \psi(x+\xi) d \xi=B(h, x) \int_{H} \psi(x+\xi) d \xi
$$

Now $\int_{I I} \psi(x+\xi) d \xi$ does not vanish since $\int_{G} \psi(x) d x$ is not zero (consider (3.2)) and so we may conclude $B(h, x)=1$ for all $h$ in $H$ and almost all $x$ in $G$.

It follows from (3.5) that $\psi$ is constant on cosets of $H$ and so we can define a unitary function $\psi_{0}$ on $G / H$ by $\psi_{0}(\pi(x))=\psi(x)$. Clearly $B_{0}$ is a coboundary determined by $\psi_{0}$. That completes the proof.

4. Projective representations and projective cocycles. Let $G$ be a locally compact Abelian group. A strongly continuous function $U$ from $G$ into the unitary operators on some Hilbert space is said to be a projective representation if

$$
U(x) U(y)=\omega(x, y) U(x+y)
$$

for some function $\omega$ of modulus one and if $U(0)=1$. We say that $\omega$ is the multiplier of the representation and it is not difficult to show that it satisfies the identity $\omega(x, y) \omega(x+y, z)=\omega(y, z) \omega(x, y+z)$ and the normalizing condition $\omega(x, 0)=\omega(0, x)=1$. Moreover, $\omega$ is continuous on $G \times G$. Conversely, given a function $\omega$ with these properties one can construct a projective representation $U_{\omega}$ with multiplier $\omega$. Indeed, define $U_{\omega}$ on $L^{2}$ by

$$
U_{\omega}(x) f(y)=\omega(x, y-x) f(y-x) .
$$

The projective representation $U_{\omega}$ is of the form

$$
U_{\omega}(x)=A_{\omega}(x, \quad) U_{0}(x)
$$

where $A_{\omega}(x, y)=\omega(x, y-x)$ is a function of modulus one on $G \times G$. The (projective) group property of $U_{\omega}$ implies that

$$
\omega(x, y) A_{\omega}(x+y, z)=A_{\omega}(x, z) A_{\omega}(y, z-\bumpeq)
$$

and the strong continuity of $U_{\omega}$ implies that $A_{\omega}(x$,$) is a strongly$ continuous operator valued function in $x$.

Observe that $A_{\omega}$ differs from the ordinary cocycle (32) in two respects; first, $A_{\omega}$ is a function on $G \times G$ instead of merely on $A \times G$, and, secondly, (4.4) replaces (2.2). We say that $A_{\omega}$ is a projective cocysle.

We say that $\omega$ is trivial if

$$
\omega(x, y)=p(x) p(y) / p(x+y)
$$

for some continuous function $p$ of modulus one on $G$. In this case any projective representation $U$ with multiplier $\omega$ can be made into an 
ordinary representation merely by multiplying $U(x)$ by $p(x)$. The product of two multipliers is again a multiplier and two multipliers whose quotient is trivial are said to be equivalent.

If $\omega$ and $\sigma$ are equivalent multipliers so that

$$
\omega(x, y) / \sigma(x, y)=p(x) p(y) / p(x+y)
$$

then a direct computation will give

$$
A_{\omega}(x, y) / A_{\sigma}(x, y)=p(x)(\varphi(y) / \varphi(y-x))
$$

where $\varphi(y)=1 / p(y)$. In particular if $\omega$ is trivial then $A_{\omega}$ is $p$ times a coboundary and conversely.

Now suppose that $G$ has a continuous one-parameter subgroup $\Lambda=\left\{e_{t} \mid t \in R\right\}$ and let $A_{\omega}$ be a projective cocycle on $G$ with $U_{\omega}$ the corresponding projective representation as given by (4.3). We wish to extract an ordinary cocycle $A$ from $A_{\omega}$ in such a way that $A$ will not be the product of a constant cocycle and a coboundary if $\omega$ is a nontrivial multiplier.

Restrict $U_{\omega}$ to $A$ so that it is a projective representation of the reals. It follows that (see the last paragraph of this section) $U_{\omega}$ is equivalent to an ordinary representation $U$ given by

$$
U\left(e_{t}\right)=p\left(e_{t}\right) U_{\omega}\left(e_{t}\right)
$$

where

$$
\omega\left(e_{t}, e_{u}\right)=p\left(e_{t}\right) p\left(e_{u}\right) / p\left(e_{t}+e_{u}\right)
$$

for some continuous function $p$ on $\Lambda$ and for all $e_{t}, e_{u} \in \Lambda$. Observe that $U$ satisfies the Weyl commutation relation

$$
U\left(e_{t}\right) V_{0}(\lambda)=\chi_{\lambda}\left(-e_{t}\right) V_{0}(\lambda) U\left(e_{t}\right)
$$

because $U_{\omega}$ does.

Consequently the operator $U\left(e_{t}\right) U_{0}\left(-e_{t}\right)$ commutes with all the $V_{0}(\lambda)$ so that

$$
U\left(e_{t}\right)=A\left(e_{t}, \quad\right) U_{0}\left(e_{t}\right), e_{t} \in \Lambda,
$$

for some ordinary cocycle $A$.

From (4.8) and (4.11) we see that

$$
A\left(e_{t}, x\right)=p\left(e_{t}\right) A_{\omega}\left(e_{t}, x\right)
$$

for all $e_{t} \in \Lambda$ and a.e. $(x)$.

We say that $A$ is the cocycle induced by $A_{\omega}$; it is uniquely determined up to a constant cocycle factor. If $A$ is the product of a constant cocycle $e^{i t \lambda}$ and a coboundary $\varphi(x) / \varphi\left(x-e_{t}\right)$ then (4.12) and (4.7) imply that $\omega$ is trivial. 
This analysis will have to be refined to yield the desired result on the torus $T^{2}$ for $T^{2}$ has no nontrivial multipliers. However, there are $\frac{1}{2} n(n-1)+1$ inequivalent local multipliers on $T^{n}$ or $R^{n}$ as shown by Bargmann [1] and local multipliers are sufficient for our purposes. Notice, in particular, that $R$ has no nontrivial local projective representations.

5. Local multipliers and cocycles on $T^{2}$. A local projective multiplier $\omega$ on the torus $T^{2}$ is a continuous function on some neighborhood $\mathscr{N} \times \mathscr{N}$ of the identity in $T^{2} \times T^{2}$ which satisfies the same functional equation and normalizing condition as a multiplier whenever $x, y$ and $x+y$ belong to $\mathscr{N}$. Unfortunately (4.3) cannot be used to define a local projective representation $U_{\omega}$, or, equivalently, a local projective cocycle $A_{\omega}$. We must resort to an ad hoc construction of $U_{\omega}$ starting from a specific nontrivial local projective multiplier $\omega$. We can then extract a cocycle from $U_{\omega}$ in much the same manner as in $\S 4$ and it is a matter of detail to prove that $A$ is not the product of a constant cocycle and a coboundary.

Let $T^{2}$ be realized as the square $[-\pi, \pi] \times[-\pi, \pi]$ with the opposite edges identified and let $\mathscr{N}$ be the open neighborhood $(-\pi, \pi) \times$ $(-\pi, \pi)$ of the identity. For a one-parameter subgroup $\Lambda$ we will take the familiar winding line with irrational slope $\alpha$.

Define $\omega$ on $\mathscr{N} \times \mathscr{N}$ by

$$
\begin{aligned}
\omega(x, y) & =\exp i\left(\left(x_{2}-\alpha^{\prime} x_{1}\right) y_{1}-\left(y_{2}-\alpha y_{1}\right) x_{1}\right) \\
& =\exp i\left(x_{2} y_{1}-y_{2} x_{1}\right)
\end{aligned}
$$

where $x=\left(x_{1}, x_{2}\right), y=\left(y_{1}, y_{2}\right)$ with $-\pi<x_{i}, y_{i}<\pi$. This is the canonical example of a nontrivial local projective multiplier on $T^{2}$ [1].

Since the complement of $\mathscr{N}$ is a null set we can regard $\omega(x, \quad)$ as a unitary function on $T^{2}$ for each fixed $x \in \mathscr{N}$. Now put $A_{\omega}(x, y)=$ $\omega(x, y-x)$ whenever $x \in \mathscr{N}$ and $y \in \mathscr{V}+x$. Then $A_{\omega}(x$,$) is a$ unitary function on $T^{2}$ for each fixed $x \in \mathscr{N}$ (the exceptional null set depends upon $x$ ). For $x \in \mathscr{N}$ we define the unitary operator $U_{\omega}(x)$ by

$$
U_{\omega}(x)=A_{\omega}(x, \quad) U_{0}(x) \text {. }
$$

It is easily verified that $U_{\omega}$ is a strongly continuous operator valued function on $\mathscr{A}$.

We will now extract a cocycle $A$ from $A_{\omega}$ even though $A_{\omega}(x$, is not defined for all $x$. The discussion parallels that of $\S 4$ and will only be given in outline.

Let $\Lambda_{1}$ denote the connected segment of $\Lambda \cap \mathscr{N}$ (relative to the ordinary real line topology on $\Lambda$ ) which contains the identity and choose a proper segment $\Lambda_{0}$ of $\Lambda_{1}$ such that $0 \in \Lambda_{0} \subseteq \Lambda_{0}+\Lambda_{0} \subseteq \Lambda_{1}$. 
For $x, y \in \Lambda_{0}, U_{\omega}$ satisfies (4.1) so that $U_{\omega}$ is a local projective representation of the reals. Consequently $U_{\omega}$ is equivalent to a local ordinary representation $U$; this means that equations (4.8) and (4.9) hold for some continuous function $p$ on $\Lambda_{1}$ (say) and for all $e_{t}, e_{u} \in \Lambda_{0}$. The local representation $U$ can be extended to a representation $U$ (keeping the same notation) of $\Lambda$ [5, Th. 63] which must satisfy the Weyl commutation relation (4.10). Exactly as before we have $U\left(e_{t}\right)=$ $A\left(e_{t},\right) U_{0}\left(e_{t}\right), e_{t} \in \Lambda$, for some ordinary cocycle $A$. We say that $A$ is the cocycle induced by $A_{\omega}$; notice that

$$
A\left(e_{t}, x\right)=p\left(e_{t}\right) A_{\omega}\left(e_{t}, x\right)
$$

holds only for $e_{t} \in \Lambda_{0}$, a.e. $(x)$.

We will now show that $A$ is not the product of a constant cocycle $C$ and a coboundary. If, on the contrary, $A$ is such a product, then

$$
A_{\omega}\left(e_{t}, x\right)=\bar{p}\left(e_{t}\right)\left(\varphi(x) / \varphi\left(x-e_{t}\right)\right)
$$

holds for all $e_{t} \in \Lambda_{0}$, a.e. (x) where we have relabeled the continuous function $C / p$ on $\Lambda_{1}$ by $\bar{p}$. In terms of the unitary operators $U_{\omega}$ and $U(y)=(\varphi() / \varphi(-y)) U_{0}(y), y \in T^{2}$, equation (5.4) becomes $U_{\omega}\left(e_{t}\right)=$ $\bar{p}\left(e_{t}\right) U\left(e_{t}\right)$ for all $e_{t} \in \Lambda_{0}$.

We wish to extend $p$ from $\Lambda_{0}$ to $\Lambda \cap \mathscr{N}$ in such a way that (5.4) remains valid. A continuity argument will then enable us to extend $p$ from $\Lambda \cap \mathscr{N}$ to $\mathscr{N}$ and this will imply that $\omega$ is trivial.

To extend $p$ from $\Lambda_{0}$ to $\Lambda \cap \mathscr{N}$ let $y \in \Lambda \cap \mathscr{N}$ so that $y \in M \Lambda_{0}=$ $\left\{M e_{t} \mid e_{t} \in \Lambda_{0}\right\}$ for some integer $M>0$. Thus $e_{t}=y M \in \Lambda_{0}$ and suppose, for the moment, that $n e_{t} \in \mathscr{N}$ for all $n \leqq M$. Then

$$
\begin{aligned}
U(y) & =U\left(M e_{t}\right)=\left(U\left(e_{t}\right)\right)^{M} \\
& =\left(p\left(e_{t}\right) U_{\omega}\left(e_{t}\right)\right)^{M} \\
& =\left[\left(p\left(e_{t}\right)\right)^{M} \prod_{k=1}^{M-1} \omega\left(e_{t}, \quad(M-k) e_{t}\right)\right] U_{\omega}(y)
\end{aligned}
$$

and we can define $p(y)$ to be the value of the expression in the brackets which obviously is independent of the representation $y=M e_{t}$.

This definition of $p(y)$ is valid whenever $(M-k) e_{t}$ is in the domain of $\omega\left(e_{t},\right)$, i.e., whenever $n e_{t} \in \mathscr{N}$ for all $0 \leqq n \leqq M$. For each $M$ there are only finitely many $y \in M \Lambda_{0}$ such that $n e_{t} \notin \mathscr{N}$ for some $0 \leqq$ $n \leqq M$. For these exceptional values we can define $p(y)$ by continuity (relative to the usual real line topology on 1 ) so that

$$
U(y)=p(y) U_{\omega}(y)
$$

holds for all $y \in \Lambda \cap \mathscr{N}$, or, equivalently, so that (5.4) holds for all $e_{t}$ in $\Lambda \cap \mathscr{N}$.

To extend $p$ from $\Lambda \cap \mathscr{N}$ to $\mathscr{N}$ we need only note that $\Lambda \cap \mathscr{N}$ is dense in $\mathscr{N}$. Let $y \in \mathscr{N}$ and choose a sequence $y_{n} \in \Lambda \cap \mathscr{N}$ which 
converges to $y$. Hence $p\left(y_{n}\right) I=U\left(y_{n}\right) U_{\omega}\left(-y_{n}\right)$ tends strongly to

$$
U(y) U_{\omega}(-y)
$$

and this limit must be of the form $p(y) I$. Alternately, $U(y) U_{\omega}(-y)$ is a multiple of the identity for each $y$ in $\mathscr{N}$ because it commutes with all bounded operators when $y$ varies over a dense subset of $\Lambda \cap \mathscr{N}$. We have now constructed a continuous function $p$ on $\mathscr{N}$ such that (5.5) holds for all $y$ in $\mathscr{N}$. Since $U_{\omega}$ is a nontrivial local projective representation of $\mathscr{N}$ this is a contradiction. Hence the induced cocycle $A$ cannot be the product of a constant cocycle and a coboundary. That completes the proof.

An interesting question remains. If $A$ is a cocycle on $T^{2}$ can one find a local projective cocycle $A_{\omega}$ which induces $A$ ? An affirmative answer should enable one to settle some of the open function theoretic questions on $T^{2}$.

The author gratefully acknowledges useful conversations with Professors F. Forelli, J. E. Gilbert and H. Helson.

\section{REFERENCES}

1. V. Bargmann, On unitary ray representations of continuous groups, Ann. of Math. 59 (1954), 1-46.

2. T. W. Gamelin, Uniform Algebras, Prentice Hall, Englewood Cliffs. N. J., 1969.

3. H. Helson, Compact groups with ordered duals, Proc. London Math. Soc. (3) 14 A (1965), 144-156.

4. H. Helson and D. Lowdenslager, Invariant subspaces, Proc. Int. Symp. on Linear Spaces, Jerusalem (1961), 251-262.

5. L. Pontrajagin, Topological Groups, Princeton, 1939.

Received April 22, 1969.

UNIVERSITY OF MONTANA

Missoula, Montana 



\section{PACIFIC JOURNAL OF MATHEMATICS}

\section{EDITORS}

H. SAMELson

Stanford University

Stanford, California 94305

R. R. PHelPS

University of Washington

Seattle, Washington 98105
J. Dugundu

Department of Mathematics

University of Southern Californle

Los Angeles, California 9.0007

RICHARD ARENS

University of California

Los Angeles, California 9.0024

\section{ASSOCIATE EDITORS}
E. F. BECKENBACH
B. H. NeumanN
F. WOLE
K. Yoshida

\section{SUPPORTING INSTITUTIONS}

UNIVERSITY OF BRITISH COLUMBIA

CALIFORNIA INSTITUTE OF TECHNOLOGY

UNIVERSITY OF CALIFORNIA

MONTANA STATE UNIVERSITY

UNIVERSITY OF NEVADA

NEW MEXICO STATE UNIVERSITY

OREGON STATE UNIVERSITY

UNIVERSITY OF OREGON

OSAKA UNIVERSITY

UNIVERSITY OF SOUTHERN CALIFORNIA

\author{
STANFORD UNIVERSITY \\ UNIVERSITY OF TOKYO \\ UNIVERSITY OF UTAH \\ WASHINGTON STATE UNIVERSITY \\ UNIVERSITY OF WASHINGTON \\ AMERICAN MATHEMATICAL SOCIETY \\ CHEVRON RESEARCH CORPORATION \\ NAVAL WEAPONS CENTER
}

The Supporting Institutions listed above contribute to the cost of publication of this Journal, but they are not owners or publishers and have no responsibility for its content or policies.

Mathematical papers intended for publication in the Pacific Journal of Mathematics should be in typed form or offset-reproduced, (not dittoed), double spaced with large margins. Underline Greek letters in red, German in green, and script in blue. The first paragraph or two must be capable of being used separately as a synopsis of the entire paper. The editorial "we" must not be used in the synopsis, and items of the bibliography should not be cited there unless absolutely necessary, in which case they must be identified by author and Journal, rather than by item number. Manuscripts, in duplicate if possible, may be sent to any one of the four editors. Please classify according to the scheme of Math. Rev. Index to Vol. 39. All other communications to the editors should be addressed to the managing editor, Richard Arens, University of California, Los Angeles, California, 90024.

50 reprints are provided free for each article; additional copies may be obtained at cost in multiples of 50 .

The Pacific Journal of Mathematics is published monthly. Effective with Volume 16 the price per volume (3 numbers) is $\$ 8.00$; single issues, $\$ 3.00$. Special price for current issues to individual faculty members of supporting institutions and to individual members of the American Mathematical Society: $\$ 4.00$ per volume; single issues $\$ 1.50$. Back numbers are available.

Subscriptions, orders for back numbers, and changes of address should be sent to Pacific Journal of Mathematics, 103 Highland Boulevard, Berkeley, California, 94708.

PUBLISHED BY PACIFIC JOURNAL OF MATHEMATICS, A NON-PROFIT CORPORATION

Printed at Kokusai Bunken Insatsusha (International Academic Printing Co., Ltd.), 7-17, Fujimi 2-chome, Chiyoda-ku, Tokyo, Japan. 


\section{Pacific Journal of Mathematics}

\section{Vol. 36, No. 2 December, 1971}

George E. Andrews, On a partition problem of H. L. Alder ............ 279

Thomas Craig Brown, An interesting combinatorial method in the theory of locally finite semigroups .......................... 285

Yuen-Kwok Chan, A constructive proof of Sard's theorem ............. 291

Charles Vernon Coffman, Spectral theory of monotone Hammerstein

operators...................................... 303

Edward Dewey Davis, Regular sequences and minimal bases .......... 323

Israel (Yitzchak) Nathan Herstein and Lance W. Small, Regular elements in

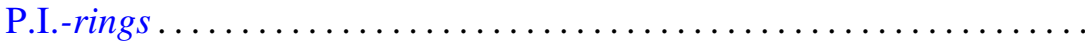

Marcel Herzog, Intersections of nilpotent Hall subgroups ..............

W. N. Hudson, Volterra transformations of the Wiener measure on the space

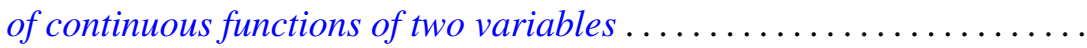

J. H. V. Hunt, An n-arc theorem for Peano spaces ................ 351

Arnold Joseph Insel, A decomposition theorem for topological group

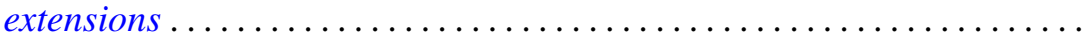

Caulton Lee Irwin, Inverting operators for singular boundary value

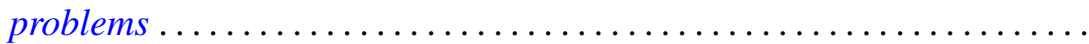

Abraham A. Klein, Matrix rings of finite degree of nilpotency ............ 387

Wei-Eihn Kuan, On the hyperplane section through a rational point of an algebraic variety...

John Hathway Lindsey, II, On a six-dimensional projective representation of $\mathrm{PSU}_{4}(3)$

Jorge Martinez, Approximation by archimedean lattice cones ...

J. F. McClendon, On stable fiber space obstructions .........

Mitsuru Nakai and Leo Sario, Behavior of Green lines at the Kuramochi boundary of a Riemann surface ....................

Donald Steven Passman, Linear identities in group rings. I. .

Donald Steven Passman, Linear identities in group rings. II ...

David S. Promislow, The Kakutani theorem for tensor products of

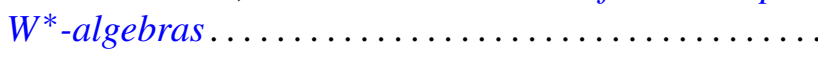

Richard Lewis Roth, On the conjugating representation of a finite group

Bert Alan Taylor, On weighted polynomial approximation of entire functions...

William Charles Waterhouse, Divisor classes in pseudo Galois

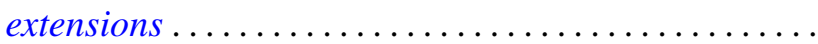

Chi Song Wong, Subadditive functions ...

Ta-Sun $\mathrm{Wu}$, A note on the minimality of certain bitransformation groups 